\title{
Automatic Detection of Arrhythmias From An ECG Signal Using An Auto-Encoder And SVM Classifier
}

\author{
Manoj Kumar Ojha ( $\square$ manojojha15@gmail.com ) \\ Madhav Institute of Technology and Science https://orcid.org/0000-0003-0557-4743 \\ Dr.Sulochna Wadhwani \\ Madhav Institute of Technology and Science \\ Dr.Arun Kumar Wadhwani \\ Madhav Institute of Technology and Science \\ Dr. Anupam Shukla \\ Indian Institute of Information Technology, Pune
}

\section{Research Article}

Keywords: ECG, Arrhythmia, Auto-encoder Convolution network, SVM, classification.

Posted Date: November 1st, 2021

DOl: https://doi.org/10.21203/rs.3.rs-981164/v1

License: (9) This work is licensed under a Creative Commons Attribution 4.0 International License.

Read Full License

Version of Record: A version of this preprint was published at Physical and Engineering Sciences in Medicine on March 18th, 2022. See the published version at https://doi.org/10.1007/s13246-022-011191. 


\title{
Automatic detection of arrhythmias from an ECG signal using an Auto-Encoder and SVM Classifier
}

\author{
Manoj Kumar Ojha ${ }^{1}$, Dr.Sulochna Wadhwani², Dr.Arun Kumar Wadhwani ${ }^{3}$, \\ Dr. AnupamShukla ${ }^{4}$ \\ 1,2,3 Madhav Institute of Technology and Science, Gwalior, Madhya Pradesh, India \\ ${ }^{4}$ Indian Institute of Information Technology, Pune,Maharashtra, India \\ EmailId:manojojha15@gmail.com ${ }^{1}$,dr.s.wadhwani@gmail.com²,wadhwani_arun@rediffmail.com ${ }^{3}$, \\ wadhwani_arun@rediffmail.com ${ }^{4}$,ORCID: https://orcid.org/0000-0003-0557-4743
}

\begin{abstract}
Millions of people worldwide are affected by arrhythmias. Arrhythmias are abnormal activity of the heart functioning. Some arrhythmias are harmful to the heart and can cause sudden mortality. The electrocardiogram (ECG) is a significant tool in cardiology for the diagnosis of arrhythmia beats. Computer-aided diagnosis (CAD) systems have been proposed in several studies to automatically classify different types of arrhythmias from ECG signals. To improve the classification of arrhythmias, a new end-to-end feature learning and classification model has been developed. This work focuses on the implementation of a one-dimensional convolution neural network (1D$\mathrm{CNN}$ ) model based on an auto-encoder convolution network (ACN) that learned the best ECG features from each heartbeat window. After that, we applied a Support Vector Machine (SVM) classifier for auto-encode features in order to detect the four different types of arrhythmic beats, including normal beats. These arrhythmia beats are left bundle branch block $(\mathrm{L})$, right bundle branch block $(\mathrm{R})$, paced beats $(\mathrm{P})$, and premature ventricular contractions $(\mathrm{V})$. using the MIT-BIH arrhythmia database. The statistical performance of the model is evaluated using tenfold crossvalidation strategies and obtained as an overall accuracy of $98.84 \%$, average accuracy of $99.53 \%$, sensitivity of $98.24 \%$ and precision of $97.58 \%$, respectively. This model has presents better results than other state-of-the-art models. Therefore, this approach may also help in clinical heart care systems.
\end{abstract}

Keywords: ECG, Arrhythmia, Auto-encoder Convolution network, SVM, classification.

\section{Introduction}

Every year, 17.9 million people are affected by cardiovascular diseases (CVD) [1]. Arrhythmia is the most common CVD. It is detected with the help of an ECG signal [2]. Arrhythmias are classified into two categories: lifethreatening and non-life-threatening [3]. Many researchers have proposed various CAD systems for detecting and classifying arrhythmia beats on small ECG datasets and achieving high performance accuracy using various feature extraction techniques $[4,5]$. There is no fully automated system for large datasets for classifying arrhythmia beats from ECG signals. The proposed end-to-end model developed for larger datasets with more accurate output may be able to help clinical experts in their analysis.

In the past few decades, ECG signals have been classified into multiple types of heart arrhythmias. Some researchers have applied machine learning (ML) techniques for the classification of ECG signals and have identified various 
types of arrhythmia beats. Before applying ML techniques, most of the studies have performed pre-processing and feature extraction tasks on ECG signals. In pre-processing steps, various types of noise are removed from the signal, while in the feature extraction step; various methods have been applied to extract suitable features from the signal database [6]. Based on these extracted features, a final ML classifier is performed to identify different types of arrhythmia beats. In recent years, Deep Learning (DL) based models have been widely used for ECG signal classification and arrhythmia identification, which perform automatic feature learning from raw ECG signal datasets [7]. Table 1 summarises some of the works that are based on ML and DL models.

Table 1 - Related work for ECG signal classification using ML and DL classifiers.

\begin{tabular}{|c|c|c|}
\hline Ref. & Author & Description \\
\hline 8 & Ye et al & $\begin{array}{l}\text { In this paper, an SVM classifier was used for the classification of } 16 \text { types of heart beats } \\
\text { from the MIT-BIH arrhythmia dataset. The morphological features and R-R interval } \\
\text { features were extracted using wavelet and independent component analysis. These } \\
\text { features were applied to SVM for the classification of } 16 \text { types of heartbeats. }\end{array}$ \\
\hline 9 & Sharma et al. & $\begin{array}{l}\text { In this study, the ECG datasets were decomposed using wavelet decomposition with a } \\
\text { dyadic orthogonal filter bank, which minimized stop-band energy and fuzzy entropy } \\
\text { features. The extract features were applied to KNN classifiers to classify five types of } \\
\text { heart beats. }\end{array}$ \\
\hline 10 & $\begin{array}{l}\text { Ebrahimnezhad } \\
\text { et al }\end{array}$ & $\begin{array}{l}\text { The ECG signal was pre-processed and a multi-resolution wavelet analysis found an R } \\
\text { peak. The QRS complex linear predictive coefficients were fed into the Probabilistic } \\
\text { Neural Network and SVM classifiers. Both classifiers were trained to test and measure } \\
\text { their performance. The experiment was conducted on the MIT-BIH data set for the } \\
\text { detection of five arrhythmia beats. }\end{array}$ \\
\hline 11 & Thomas et al & $\begin{array}{l}\text { The multilayer back propagation neural network classifier was used to classify five } \\
\text { different types of arrhythmia beats from the MIT-BIH arrhythmia dataset. Classification } \\
\text { was performed on extracted features based on discrete wavelet transform and dual tree } \\
\text { complex wavelet transform. }\end{array}$ \\
\hline 12 & Pandey et al & $\begin{array}{l}\text { This research presents feature extraction methods using higher-order statistics, wavelets, } \\
\text { morphological descriptors, and R-R intervals. These features were fed into a long short- } \\
\text { term memory network model for classification. The MIT-BIH arrhythmia datasets were } \\
\text { used for training and testing of models for classifying five different types of arrhythmia } \\
\text { beats. }\end{array}$ \\
\hline 13 & Nurmaini et al & $\begin{array}{l}\text { A deep neural network was used for classifying ten different types of arrhythmia beats on } \\
\text { the basis of deep auto-encoder extracted features from the MIT-BIH arrhythmia dataset. In } \\
\text { addition, DL enables embedded feature extraction and selection directly from raw ECG } \\
\text { data during the pre-training and fine-tuning processes of the deep auto-encoder and deep } \\
\text { neural network. }\end{array}$ \\
\hline
\end{tabular}




\begin{tabular}{l|l|l}
\hline 14 & Hong et al & $\begin{array}{l}\text { This paper suggests a two-stage approach consisting of feature extraction and classifier } \\
\text { development. As part of the feature extraction procedure, both engineered and DL features } \\
\text { were retrieved. The authors discuss feature aggregation methods for combining these } \\
\text { features and creating fixed-dimensional feature vectors. In the second step, they generate } \\
\text { multiple gradient-boosting decision trees and combine them to obtain the final } \\
\text { classification. }\end{array}$ \\
\hline 15 & Yang et al & $\begin{array}{l}\text { Presented an ECG arrhythmia classification technique based on the softmax regression } \\
\text { model. The stacked sparse auto-encoders extracted features from the MIT BIH arrhythmia } \\
\text { data set, which was shown to be efficient in extracting 'in-depth' features. }\end{array}$ \\
\hline 16 & Yildirim et al & $\begin{array}{l}\text { The author proposed a 1D convolutional neural network model for classifying arrhythmia } \\
\text { beats into 17 classes. An end-to-end model was established in this model to analyse 10- } \\
\text { second long-duration ECG signal fragments from one lead of 45 subjects from the MIT- } \\
\text { BIH arrhythmia database. }\end{array}$ \\
\hline
\end{tabular}

In this paper, we have proposed a1D-CNN model as an auto-encoder that encodes and learns the best features from the ECG dataset. These encoded features have been applied to the SVM classifier, which identify the arrhythmia beats from the dataset. There is no need of handcrafted feature extraction methods from the ECG signal. The performance of the model is calculated in terms of accuracy, precision and recall and achieved results are better compared to the previous works.

\section{Material and method}

The proposed work flow diagram of the ECG signal classification to detect arrhythmia from the MIT-BIH arrhythmia dataset is given in figure 1.

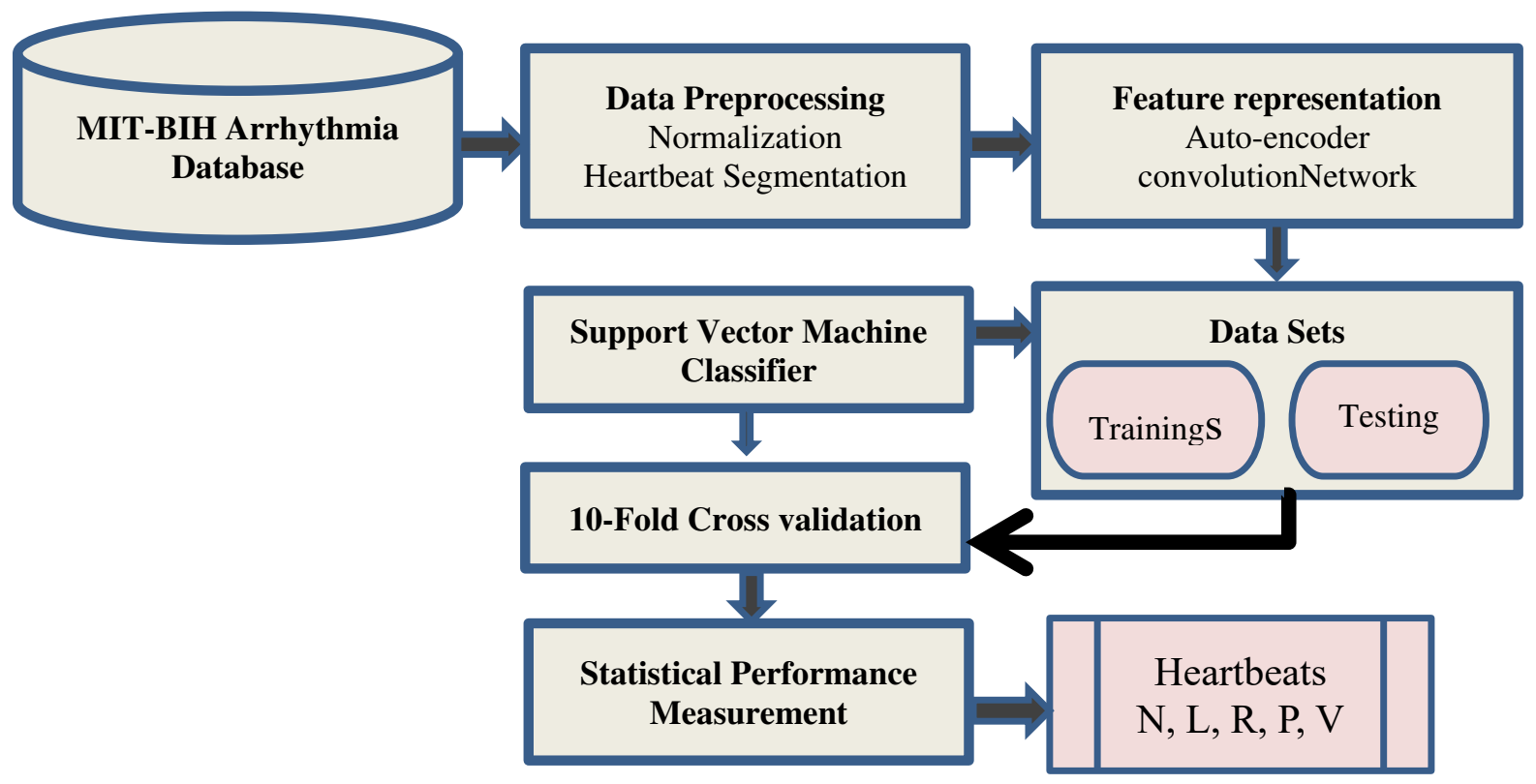

Fig 1: Proposed workflow diagram for ACN based SVM classification Model. 
In this study, the first dataset is normalised using the Z-score normalisation method. After normalisation, each patient record dataset is segmented into heartbeats based on the annotation file given by the cardiologist. The segmented dataset is fed into the auto-encoder based convolution neural network, which learns the ECG signal characteristics. Finally, an SVM classifier is used to identify the five types of arrhythmias.

\subsection{Database Used:}

For the life-threatening arrhythmia beat detection of the proposed study, the MIT-BIH arrhythmia ECG dataset is used which has been taken from open source available from the PhysioNet website [17]. It contains 48 records of cardio patients, each of which contains reference annotation files for each beat. The 100 series has 23 recordings from a routine clinical recording of a person, whereas the 200 series contains recordings from an unusual but clinically significant case of arrhythmias that were not adequately shown in the 100 series recordings. Each record is approximately 30 minutes long and each heartbeat's label by two or more cardiologists is annotated to indicate the class of heartbeat [18]. Figure 2 shows is a graphical representation of the five different types of ECG signals.
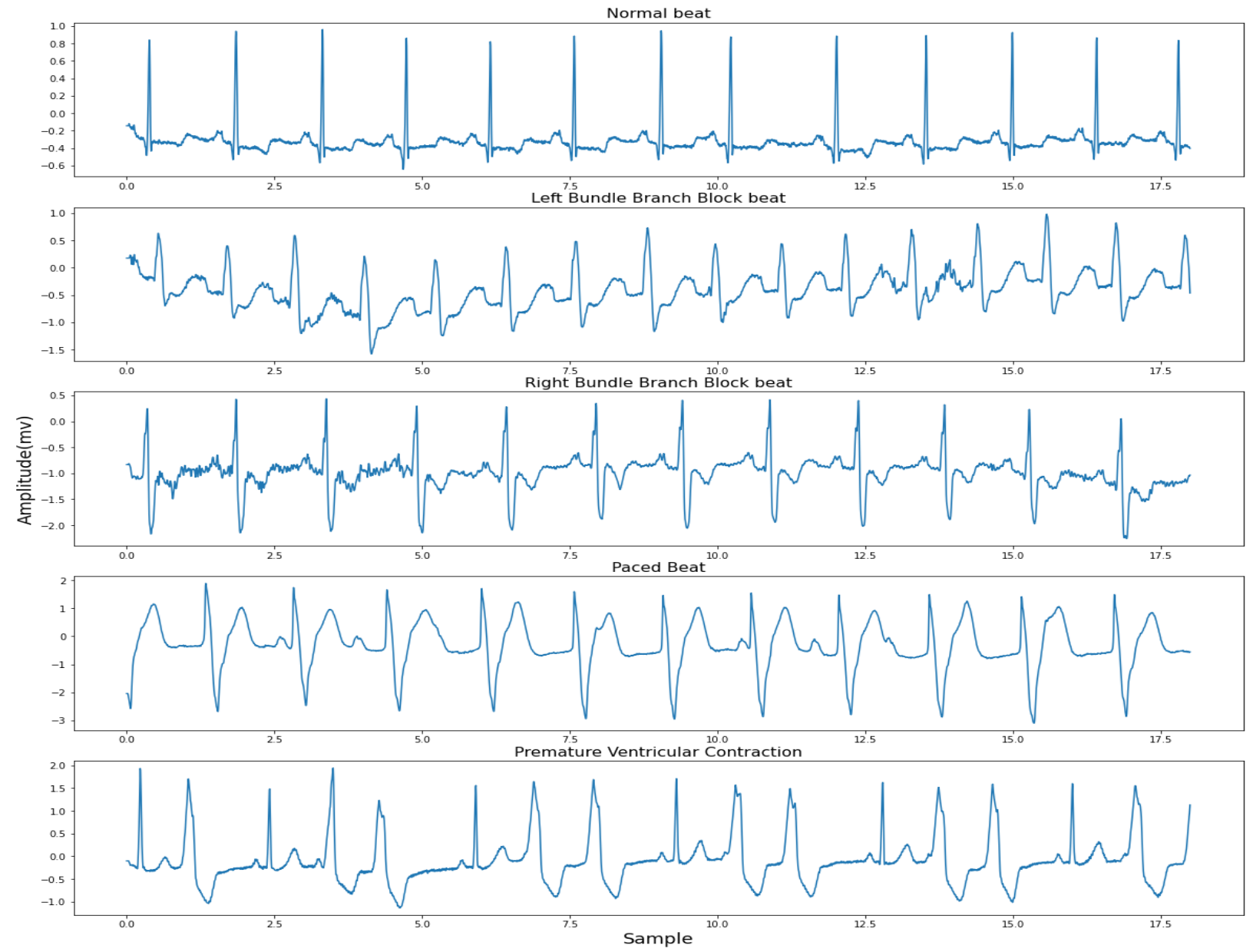

Figure 2: Typical ECG beats of (a) Normal, (b) LBBB, (c) RBBB, (d) PB, (e) PVCs. 
Fig. 2(a) shows the typical cardiac beat when the heart is functioning normally. If there is an abnormality in the conduction of the electrical pulse generated by the SA node in the left/right bundle branch, depolarization is delayed. It is composed of two variants of the bundle branch block. They are classified as LBBB and RBBB. LBBB is referred to as a problem detected on the left side of the heart and maybe visualized by a wide QRS complex in Fig. 2(b). In fig. 2(c), and show that RBBB is a problem on the right side of the heart and that the QRS complex is of a large size. The PB is an atrial paced rhythm that occurs when the atria are paced but not the ventricles. Fig. 2(d) shows the QRS complex is narrow in size. PVCs are very dangerous compared to other types of heart rhythms. The next cardiac cycle is enlarged than usual, as shown in fig. 2 (e).

\subsection{Normalization and heartbeat Segmentation}

The technique of normalisation centred values around a mean with a unit standard deviation. It means if we calculate the mean and standard deviation of normalised scores, they will be 0 and 1 respectively. The $\mathrm{Z}$ score is determined [19]; it is defined by the equation.

$$
Z=\frac{(X-\mu)}{\sigma}
$$

Where, $\mathrm{X}=$ Input variable, $\mu=$ Mean of the distribution, $\sigma=$ standard deviation of distribution.

To obtain different types of heartbeats, the ECG signal was split into 300 sample chunks based on the annotation file given in the MIT BIH database. The 300 samples are selected because the arrhythmia class is much faster than normal heartbeats. In the segmentation process [19], the position of the QRS complex's R peak is first determined using annotation file information, and then 149 sample points are taken before and 150 sample points after the $\mathrm{R}$ peak, respectively 300 points after the $\mathrm{R}$ peak ensures that one beat is present in a single chunk, and the central beat must be classified against five possible arrhythmia labels. Similarly, all ECG signals are segmented.

\subsection{Proposed Deep Convolution Auto-encoded Network (ACN)}

The 1D-auto-encoder convolution is capable of encoding data at the input layer and reconstructing it at the output layer, with the objective of reproducing the input signal as similar as possible. This is accomplished by training the neural network to change the input data while ensuring that the relevant information is not lost in the encoding phase [20-21]. This procedure enables the machine to generate a feature extraction mechanism by identifying the most relevant parameters in the ECG signal. Let's consider the input vector of auto-encoder $x \in[0,1]^{d}$ and it maps to hidden representation $y \in[0,1]^{d}$ between input-output mappings.

$$
\begin{aligned}
& y=f_{a}(x)=\operatorname{ReLu}\left(W_{x}+b\right) \\
& \text { ReLu }=\left\{\begin{array}{c}
0, \text { if } x<0 \\
x, \quad \text { otherwise }
\end{array}\right.
\end{aligned}
$$

Where, ReLu(Rectified Linear Unit $)=\max (0, x)$ and $W$ is a weight matrix of $d X d$ and $b$ is a bias vector and $\operatorname{ReLu}$ is activation function. The hidden output $y$ is again mapped to back to re-produced vector $\in[0,1]^{d}$. Here, the 
ACN keeps on learning to get the best weights. This is done by feedback sent from output periodically to update weights until the best combination is found. ACN's main objective is to compress each heartbeat segment with the smallest losses possible. To achieve this objective, an $\mathrm{ACN}$ has been designed to compress the segmented data and extract auto-encoded features from each encoded heartbeat window.

Table 2 : Deep ACN architecture for feature encoder from ECG signal.

\begin{tabular}{|c|c|c|c|c|}
\hline Layers & Layer types & Output neurons & Kernel Size & Stride \\
\hline 1 & Convolution & $281 \times 3$ & 20 & 1 \\
\hline 2 & Max-pooling & $140 \times 3$ & 2 & 2 \\
\hline 3 & Convolution & $131 \times 5$ & 10 & 1 \\
\hline 4 & Max-pooling & $65 \times 5$ & 2 & 2 \\
\hline 5 & Convolution & $63 \times 10$ & 3 & 1 \\
\hline 6 & Max-pooling & $31 \times 10$ & 2 & 2 \\
\hline 7 & Convolution & $31 \times 4$ & 1 & 1 \\
\hline 8 & Max-pooling & $15 \times 4$ & 2 & 2 \\
\hline 9 & $\begin{array}{c}\text { Flatten } \\
\text { (Encoded features) }\end{array}$ & 60 & -- & -- \\
\hline
\end{tabular}

The auto encoder has four convolution layers and four max pooling layers for extracting features by convolving the input features produced by the intermediate layer link convolution and max pooling. The function of ReLU is to map a negative value to zero and hence have all positive values so that the training can be fast and more effective. The activation output of this layer has been normalized using the batch normalisation layer. Here we take the input length 300 weight vector. These weighted vectors are given to the first convolution layer with filter size 20 . This is also known as the kernel size. After convolution, apply the max pooling layer with a $(2,2)$ filter and stribe 2 . The pooling layers generally reduce the size of feature vectors. This indicates that the output matrix is just half the size of the input matrix. The batch normalization layer is used to normalize the activation output of the max-pooling layer. Similarly, the $3^{\text {rd }}, 5$ th, and $7^{\text {th }}$ convolution processes are performed correspondingly using max pooling and batch normalisation layers, and the filter sizes for each convolution layer are 10, 3, and 1. Table 3 gives the illustration description of each convolution, max pooling, and batch normalisation layer output. After the last layer, 
we used a flattening layer which gives the 60 encoded features of each heartbeat segment. These features are given to the SVM classifier for classification of arrhythmia beats.

\subsection{Support Vector Machine Classifier}

SVM is based on the concept of finding the optimal hyperplane between data values corresponding to distinct classes [22-23].In the case of two classes, $f(x)>0$ i.e $\{+1\}$ and $f(x)<0$ i.e $\{-1\}$, the goal is to find a separating hyper plane. This mapping can be done by either linear or nonlinear kernel functions. This hyper plane is defined as

$$
w^{\prime} \phi(x)+b=0
$$

Where, $X=\left(x_{1}, x_{2}, \ldots \ldots . x_{d}\right)$ is the vector of predictors and $d$ is dimension. This vector is mapped into a higher dimension feature space by a non-linear $\phi$, a vector $\mathrm{W}$ of weights and $a$ bias $b$. The aim of training is to find the optimal hyperplane. This can be achieved by maximizing the distance of separation between the two planes $w^{\prime} \phi(x)+b=-1$ and $w^{\prime} \phi(x)+b=+1$.For a given set of learning $\operatorname{data}\left(x_{i}, y_{i}\right)$ for $i=1,2, \ldots \ldots \ldots \ldots m$, the formulation of optimization issue for the SVM is given as:

$$
\min _{w, \xi, b} J(w, \xi)=\frac{1}{2} w^{\prime} w+c \sum_{j=1}^{n} \xi_{j}
$$

Such that

$$
y_{j}\left(w^{\prime} \phi(x,)+b\right) \geq 1-\xi_{j}, j=1,2, \ldots \ldots \ldots, \xi_{j} \geq 0,
$$

Where $\mathrm{c}$ is a positive regularisation constant chosen by observation, $\mathrm{W}$ is a weight vector for learning parameters, $\xi_{j}$ is a positive slack variable indicating the distance of $x_{j}$ from the decision boundary, and $\varphi$ is a nonlinear mapping function applied to map input data point $x_{j}$ into a greater dimensional space. The SVM may be represented using lagrange multipliers $\alpha \geq 0$. The response to the lagrange multipliers problem is found by solving a quadratic programming problem [35]. The SVM decision function can be given as

$$
g(x)=\sum_{x_{j} \in S V}^{n} \alpha_{j} y_{j} K\left(x_{i}, x_{j}\right)+b
$$

The kernel is the radial basis function (RBF)

$$
K\left(X_{i}, X_{j}\right)=e^{-\left(\frac{\left\|X_{i}-X_{j}\right\|}{2 \sigma^{2}}\right)}
$$

Here, the RBF kernel is used as an SVM kernel function. All ECG segments that have a different type of heartbeat are mapped to the feature space using the ACN that has been explained above. The above encoded features obtained by the ACN model are divided into two parts: training and validation. First, the SVM model has been trained on a training set of $90 \%$ of the dataset set from total features, and remaining $10 \%$ of the dataset as a validation dataset has been fitted to the SVM model to detect arrhythmias in heartbeats.

\section{Experimental Result}

We used ECG data from the MIT-BIH Arrhythmia Database, which included data for the normal heartbeat as well as data for four different forms of arrhythmias to perform the statistical measures. The training used a total of 88075 data points. There are still 9786 data points to test. The parameters ( $c$ and $y$ ) of the SVM classification should be 
adjusted in order to obtain the most optimal classification performance. The value of $y$ is important for the classification effect. Therefore, we must first determine the optimal parameter candy using ten-fold cross-validation techniques. We split the entire data set into ten equal sections using ten-cross validation strategies. Out of the ten sections, nine are used for training and the remaining one is a test for model validation. This process is repeated ten times, where each time a different test set is used to validate the model. The test samples are those samples of the data set which have been seen in the training phase. Thus, ten-fold validation is performed on the dataset. Based on the accuracy; we have identified the best parameters. The average of ten-fold SVM classification results for all classes of heartbeats are shown in the confusion matrix. This experiment used an SVM classifier to classify heart rhythms into five separate classes based on training and testing data.

Table 3: The average of all 10-fold confusion matrix for the validation dataset.

\begin{tabular}{|c|c|c|c|c|c|}
\hline \multirow{2}{*}{ Heartbeat Type } & \multicolumn{5}{|c|}{ Predicted Heartbeat } \\
\cline { 2 - 6 } & $\mathbf{N}$ & $\mathbf{L}$ & $\mathbf{R}$ & $\mathbf{P}$ & $\mathbf{V}$ \\
\hline $\mathbf{N}$ & 7141 & 7 & 4 & 0 & 17 \\
\hline $\mathbf{L}$ & 10 & 779 & 0 & 0 & 5 \\
\hline $\mathbf{R}$ & 10 & 1 & 701 & 0 & 0 \\
\hline $\mathbf{P}$ & 0 & 0 & 0 & 389 & 1 \\
\hline $\mathbf{V}$ & 35 & 17 & 5 & 1 & 662 \\
\hline
\end{tabular}

The diagonal components represent the beats that have been accurately identified as belonging to their respective classes. On the basis of the confusion matrix for the validated dataset, it is clear that $28 \mathrm{~N}$ beats, $15 \mathrm{~L}$ beats, $11 \mathrm{R}$ beats, $1 \mathrm{P}$ beat, and $58 \mathrm{~V}$ beats are misclassified.

\subsection{Performance Evaluation Metrics:}

The proposed model has been trained and validated on a desktop Intel i-5 CPU with 8 GB RAM. The Keras and Tensorflow packages are used with the Python programming language.

Table 4: ECG classification parameters are being evaluated for performance measurement.

\begin{tabular}{|ccl|}
\hline Parameter & Formula & \multicolumn{1}{c|}{ Definition } \\
\hline Precision (Pe) & $\frac{T P}{T P+F P}$ & The proportion of true positive among all classified positive. \\
Sensitivity (Se) & $\frac{T P}{T P+F N} * 100$ & The proportion of true positive among the entire set of positive. \\
Accuracy (Ac) & $\frac{T P+T N}{T P+F N+F P+T N} * 100$ & $\begin{array}{l}\text { The widely used metric for determining the overall model } \\
\text { performance. }\end{array}$ \\
& & \\
\end{tabular}


Table 4 illustrates how the values of TP, TN, FN, and FP have been used to determine evaluation parameters. On the basis of confusion matrix parameters such as true positive (TP), false positive (FP), true negative (TN), and false negative (FN) values, the model's performance is evaluated in terms of accuracy, sensitivity, and precision [36].

The statistical performance indicates the proposed end-to-end model for five classes are summarized in table 5, which contains the sensitivity, precision, and accuracy. The experimental results indicate that the proposed method has the capability to classify the heartbeats into five different classes with an overall accuracy of $98.84 \%$ using SVM.

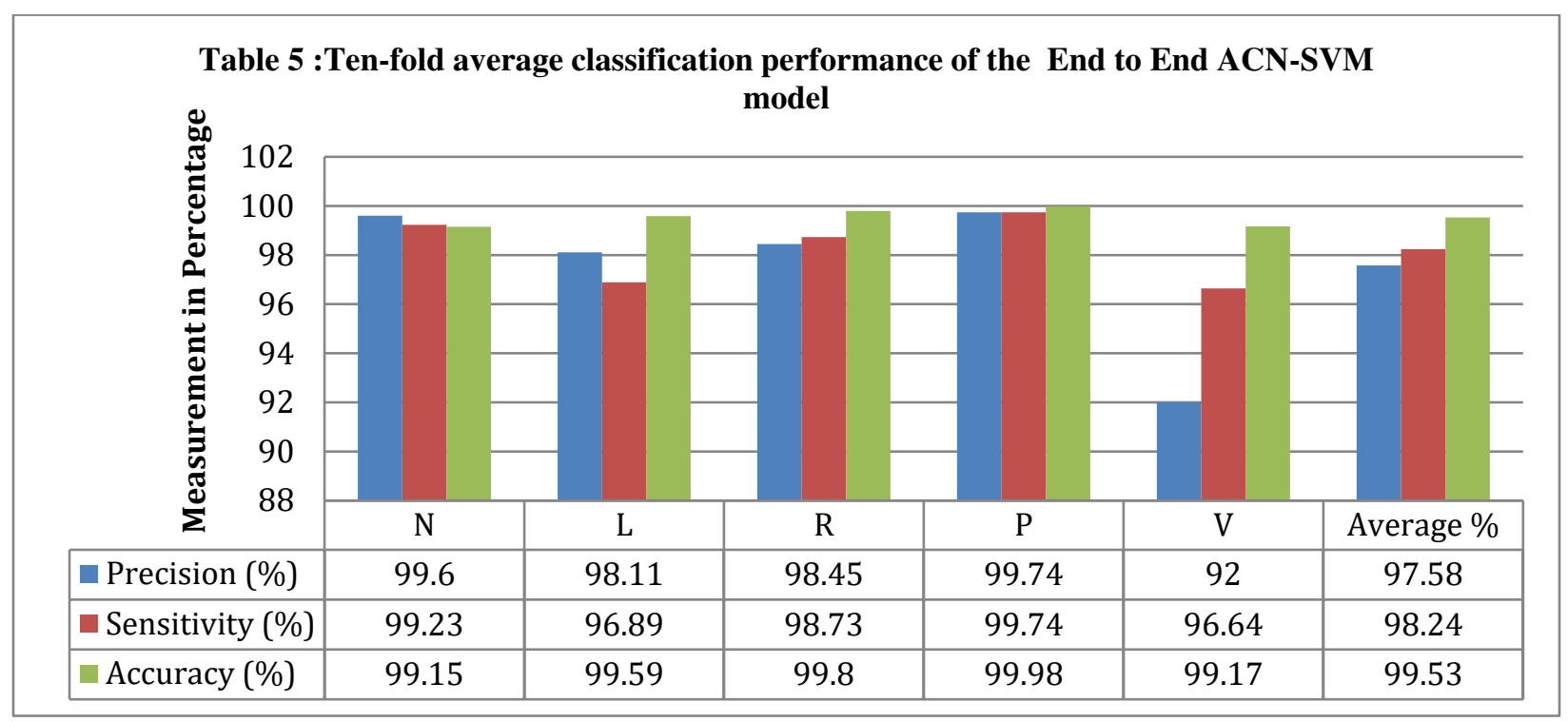

The precision is $97.58 \%$, the average accuracy is $99.53 \%$, the sensitivity is $98.24 \%$, and the overall accuracy is 98.84\%.The advantage of developing this model is that it is an end-to-end classifier that does not require de-noising of the ECG signal. The results demonstrate excellent classification accuracy, indicating that the proposed method has the potential to become an effective way for cardiologists to detect arrhythmia using ECG signals.

\section{Discussion}

There are several research studies in the literature based on the classification problem of ECG data that have been obtained from the publically available MITBIH arrhythmia database. The classification performance of the proposed system is compared with the state-of-the-art approach in Table 6. Li et al. [24] performed feature extraction and classification of six different types of heartbeats using a genetic algorithm back propagation neural network for classification and feature extraction using wavelet packet decomposition. According to statistics, the result achieved a classification accuracy of $97.78 \%$ for 6 different classes. Sahoo et al. [25] proposed a multi wavelet transform feature set that accurately identifies four types of ECG beats: normal, LBBBs, RBBBs, and Paced beats using SVM classification on the MIT-BIH ECG database, achieving 98.39\% accuracy. Jung et al. [26] have used integrated PCA and LDA features and used fitness rules and a weighted KNN algorithm to classify four different types of arrhythmia heartbeats based on the combined features. The results indicate a sensitivity of $97.57 \%$. 
Table 6: Comparison between the related work on MIT-BIH ECG database and the method proposed in this work.

\begin{tabular}{|c|c|c|c|c|c|}
\hline Works\& Year & Features Extraction & Classifier & $\begin{array}{c}\text { Accuracy } \\
\%\end{array}$ & $\begin{array}{c}\text { Sensitivity } \\
\%\end{array}$ & $\begin{array}{c}\text { Precision } \\
\%\end{array}$ \\
\hline $\begin{array}{c}\text { Li et al. [24] } \\
\text { 2017 }\end{array}$ & $\begin{array}{c}\text { Statistical features of } \\
\text { WPCs }\end{array}$ & GA-BPNN & 97.78 & 99.33 & 99.36 \\
\hline $\begin{array}{c}\text { Sahoo et al.[25] } \\
\text { 2017 }\end{array}$ & DWT, Temporal & SVM & 98.39 & 96.89 & - \\
\hline $\begin{array}{c}\text { Jung et al.[26] 2017 } \\
\text { DWT, PCA, LDA }\end{array}$ & WKNN & 96.12 & 96.12 & - \\
\hline Oh et al. [27] 2018 & End to End DL model & CNN-LSTM & 98.1 & 98.7 & 97.5 \\
\hline Wu et al.[7] 2021 & Wavelet & CNN & 97.41 & 97.05 & - \\
\hline Atal et.al [28] 2020 & Gabor Filter, Wavelet & $\begin{array}{c}\text { BaRoA- } \\
\text { DCNN }\end{array}$ & 93.19 & 93.98 & \\
\hline Proposed & ACN features & SVM & 99.53 & 98.24 & \\
\hline
\end{tabular}

Atal et al. [28] developed a deep convolutional neural network based on optimization that obtained $93.19 \%$ accuracy across five classes. The experimental results show the precision is $97.58 \%$, the average accuracy is $99.53 \%$, the sensitivity is $98.24 \%$, and the overall accuracy is $98.84 \%$ in the classification of five different ECG arrhythmias.

\section{Conclusion:}

The ECG signal abnormality analysis was explained and proposed in this paper. This research was focused on the classification of Arrhythmia heartbeats. Classifiers were employed in the MIT-BIH Arrhythmia Database to identify four types of arrhythmia heartbeats and normal heartbeat. The SVM was trained using extracted features from the ACN model and then validated on a test data set by applying 10-fold class validation strategies for the classification of the arrhythmia heartbeats. Computer simulations have shown that our approach provides excellent performance, with $98.84 \%$ over all accuracy and average accuracy of $99.53 \%$ respectively. It may be concluded that the model can be a powerful cardiac diagnostic system for automatic arrhythmia detection without using handcrafted features. 
Conflict of Interest: Author Manoj Kumar Ojha declares that he/she has no conflict of interest. Author Dr.

Sulochna Wadhwani declares that he/she has no conflict of interest. Author Dr. Arun Kumar Wadhwani declares that he/she has no conflict of interest. Author Dr. Anupam Shukla declares that he/she has no conflict of interest.

\section{Ethical approval:}

This article does not contain any studies with human participants or animals performed by any of the authors.

\section{Reference:}

[1]. World Health Organization, "Cardiovascular diseases (CVDs)," 2017. [Online]. Available: http://www.who.int/mediacentre/factsheets/fs317/en/. [Accessed: 2020].

[2]. The European Society for Cardiology. ESC Guidance for the Diagnosis and Management of CV Disease during the COVID-19 https://www.escardio.org/Education/COVID-19and-Cardiology/ESCCOVID-19Guidance. (Accessed: 2020).

[3]. National Heart Lung and Blood Institute, "Types of arrhythmias," 2011. [Online]. Available: https://www.nhlbi.nih.gov/health/health-topics/topics/arr/types. [Accessed: 2020].

[4]. Singh, Shraddha, et al. "Classification of ECG arrhythmia using recurrent neural networks." Procedia computer science 132 (2018): 1290-1297. https://doi.org/10.1016/j.procs.2018.05.045

[5]. Acharya, U. Rajendra, et al. "A deep convolutional neural network model to classify $\begin{array}{llllll}\text { heartbeats." Computers in } \quad \text { biology and } & \text { 389-396. }\end{array}$ https://doi.org/10.1016/j.compbiomed.2017.08.022

[6]. Yanase, Juri, and EvangelosTriantaphyllou. "A systematic survey of computer-aided diagnosis in medicine: Past and present developments." Expert Systems with Applications 138 (2019): 112821. https://doi.org/10.1016/j.eswa.2019.112821

[7]. Wu, Mengze, et al. "A Study on Arrhythmia via ECG Signal Classification Using the Convolutional Neural Network." Frontiers in computational neuroscience 14 (2020): 106. DOI: 10.3389/fncom.2020.564015

[8]. Ye, Can, BVK Vijaya Kumar, and Miguel Tavares Coimbra. "Heartbeat classification using morphological and dynamic features of ECG signals." IEEE Transactions on Biomedical Engineering 59.10 (2012): 29302941. DOI: $10.1109 /$ TBME.2012.2213253

[9]. Sharma, Manish, Ru-San Tan, and U. Rajendra Acharya. "Automated heartbeat classification and detection of arrhythmia using optimal orthogonal wavelet filters." Informatics in Medicine Unlocked 16 (2019): 100221. https://doi.org/10.1016/j.imu.2019.100221

[10]. EBRAHIMNEZHAD, Hossein, and Shiva KHOSHNOUD. "Classification of arrhythmias using linear predictive coefficients and probabilistic neural network." Applied Medical Informatics. 33.3 (2013): 55-62. https://ami.info.umfcluj.ro/index.php/AMI/article/view/434

[11]. Thomas, Manu, Manab Kr Das, and Samit Ari. "Automatic ECG arrhythmia classification using dual tree complex wavelet based features." AEU-International Journal of Electronics and Communications 69.4 (2015): 715-721. https://doi.org/10.1016/j.aeue.2014.12.013

[12]. Pandey, Saroj Kumar, and Rekh Ram Janghel. "Automatic arrhythmia recognition from electrocardiogram signals using different feature methods with long short-term memory network model." Signal, Image and Video Processing 14.6 (2020): 1255-1263. https://doi.org/10.1007/s11760-020$\underline{01666-8}$

[13]. Nurmaini, Siti, et al. "An automated ECG beat classification system using deep neural networks with an unsupervised feature extraction technique." Applied Sciences 9.14 (2019): 2921. https://doi.org/10.3390/app9142921 
[14]. Hong, Shenda, et al. "Combining deep neural networks and engineered features for cardiac arrhythmia detection from ECG recordings." Physiological measurement $40.5 \quad$ (2019): 054009. https://doi.org/10.1088/1361-6579/ab15a2

[15]. Yang, Jianli, et al. "A novel electrocardiogram arrhythmia classification method based on stacked sparse auto-encoders and softmax regression." International Journal of Machine Learning and Cybernetics 9.10 (2018): 1733-1740. https://doi.org/10.1007/s13042-017-0677-5

[16]. Yıldırım, Özal, et al. "Arrhythmia detection using deep convolutional neural network with long duration ECG signals." Computers in biology and medicine 102 (2018): 411-420. https://doi.org/10.1016/j.compbiomed.2018.09.009

[17]. Goldberger, Ary L., et al. "PhysioBank, PhysioToolkit, and PhysioNet: components of a new research resource for complex physiologic signals." circulation 101.23 (2000): e215-e220. https://doi.org/10.1161/01.CIR.101.23.e215

[18]. Moody, George B., and Roger G. Mark. "The impact of the MIT-BIH arrhythmia database." IEEE Engineering in Medicine and Biology Magazine 20.3 (2001): 45-50. DOI: 10.1109/51.932724

[19]. Xu, Sean Shensheng, Man-WaiMak, and Chi-Chung Cheung. "Towards end-to-end ECG classification with raw signal extraction and deep neural networks." IEEE journal of biomedical and health informatics 23.4 (2018): 1574-1584. DOI: 10.1109/JBHI.2018.2871510

[20]. LeCun, Yann, YoshuaBengio, and Geoffrey Hinton. "Deep learning." nature 521.7553 (2015): 436-444. https://doi.org/10.1038/nature14539

[21]. Goodfellow, Ian, YoshuaBengio, and Aaron Courville. Deep learning. MIT press, 2016.

[22]. Li, Duan, Hongxin Zhang, and Mingming Zhang. "Wavelet de-noising and genetic algorithm-based least squares twin SVM for classification of arrhythmias." Circuits, Systems, and Signal Processing 36.7 (2017): 2828-2846. https://doi.org/10.1007/s00034-016-0439-8

[23]. de Albuquerque, Victor Hugo C., et al. "Robust automated cardiac arrhythmia detection in ECG beat signals." Neural Computing and Applications 29.3 (2018): 679-693. https://doi.org/10.1007/s00521-016$2472-8$

[24]. Li, Hongqiang, et al. "Genetic algorithm for the optimization of features and neural networks in ECG signals classification." Scientific reports 7.1 (2017): 1-12. DOI: 10.1038/srep41011

[25]. Sahoo, Santanu, et al. "Multiresolution wavelet transform based feature extraction and ECG classification to detect cardiac abnormalities." Measurement 108 (2017): 55-66. https://doi.org/10.1016/j.measurement.2017.05.022

[26]. Jung, W-H., and S-G. Lee. "An arrhythmia classification method in utilizing the weighted KNN and the fitness rule." IRBM 38.3 (2017): 138-148. https://doi.org/10.1016/j.irbm.2017.04.002

[27]. Oh, ShuLih, et al. "Automated diagnosis of arrhythmia using combination of CNN and LSTM techniques with variable length heart beats." Computers in biology and medicine 102 (2018): 278-287. https://doi.org/10.1016/j.compbiomed.2018.06.002

[28]. Atal, Dinesh Kumar, and Mukhtiar Singh. "Arrhythmia classification with ECG signals based on the optimization-enabled deep convolutional neural network." Computer Methods and Programs in Biomedicine 196 (2020): 105607. https://doi.org/10.1016/j.cmpb.2020.105607 